Bundesgesundheitsbl 2015 · 58:505-512

DOI 10.1007/s00103-015-2132-7

c) Springer-Verlag Berlin Heidelberg 2015

Bekanntmachung des Umweltbundesamtes

\title{
Richtwerte für Butanonoxim in der Innenraumluft
}

\section{Mitteilung der Ad-hoc-Arbeitsgruppe Innenraumrichtwerte der Kommission Innenraumlufthygiene und der Obersten Landesgesundheitsbehörden}

CAS-Nummer: 96-29-7

Summenformel: $\mathrm{C}_{4} \mathrm{H}_{9} \mathrm{NO}$

Strukturformel:

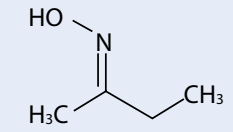

(E-Form)

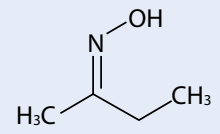

(Z-Form) zum einen ein Wirkungsmechanismus vorliegt, der einen Schwellenwert begründet, und zum anderen die Kanzerogenität nicht den empfindlichsten Wirkendpunkt darstellt. Diese Voraussetzung trifft für Butanonoxim zu. Demnach stellt die Degeneration des olfaktorischen Epithels der Maus den empfindlichsten Wirkendpunkt von Butanonoxim dar. Diese Wirkung tritt bei einer Konzentration auf, die um mehr als eine Größenordnung unterhalb der bei der Ratte beobachteten krebserzeugenden Wirkungskonzentration von Butanonoxim liegt. Angesichts des Fehlens belastbarer Humandaten geht die Ad-hoc-Arbeitsgruppe für ihre Ableitung von Richtwerten für Butanonoxim in der Innenraumluft von tierexperimentellen Daten aus.

\section{Stoffidentifizierung}

Systematischer Name: Butanonoxim Synonyme: 2-Butanonoxim, Ethylketoxim, Ethylmethylketoxim, MEK-Oxim, MEKO, Methylethylketonoxim, Methylethylketoxim

CLP-Index-Nr.: 616-014-00-0

EG-Nummer: 202-496-6

\subsection{Physikalische und chemische Eigenschaften}

Butanonoxim ist ein kurzkettiges Ketoxim, das zur chemischen Klasse der Oxime gehört. Oxime entstehen bei der Reaktion von Aldehyden oder Ketonen mit Hydroxylaminen.

Molekularmasse: $87,12 \mathrm{~g} / \mathrm{mol}$

Schmelzpunkt: $-29,5^{\circ} \mathrm{C}$

Siedepunkt: $152^{\circ} \mathrm{C}(1013 \mathrm{hPa})$

Dichte: $920 \mathrm{~kg} / \mathrm{m}^{3}$ bei $20^{\circ} \mathrm{C}$

Dampfdruck: $1,41 \mathrm{hPa}$ bei $20^{\circ} \mathrm{C}$

Wasserlöslichkeit: $114 \mathrm{~g} / \mathrm{l}$ bei $20^{\circ} \mathrm{C}$

Umrechnung (bei $20^{\circ} \mathrm{C}, 1013 \mathrm{hPa}$ ): $1 \mathrm{mg} /$ $\mathrm{m}^{3}=0,276 \mathrm{ml} / \mathrm{m}^{3} ; 1 \mathrm{ml} / \mathrm{m}^{3}=3,62 \mathrm{mg} / \mathrm{m}^{3}$

\subsection{Stoffeigenschaften und Anwendung}

Butanonoxim ist eine farblose bis leicht gelbliche, leicht wasserlösliche, unangenehm aromatisch riechende Flüssigkeit mit mäßiger Flüchtigkeit. Beim Erhitzen können sich mit Luft explosive Dämpfe entwickeln.
Butanonoxim wird u. a. zur Vermeidung der Oberflächenhautbildung bei Alkydharzen, die Bestandteile von Lacken, Beschichtungen und Druckfarben etc. sind, eingesetzt. Eine wichtige Quelle für die Exposition gegenüber Butanonoxim können daher Anstrichfarben, aber auch Fugenmassen sein [3].

\section{Exposition}

\subsection{Innenraumluft}

Relevanter Expositionspfad von Butanonoxim für die Allgemeinbevölkerung ist die inhalative Aufnahme in Innenräumen. Die US-amerikanische Umweltschutzbehörde (US-EPA) beschreibt in ihrem „EPA's Indoor Air Quality Research Update" [4] Messmethoden und Ergebnisse $\mathrm{zu}$ flüchtigen organischen Komponenten und gefährlichen Luftverunreinigungen aus Alkyd- und LatexFarben. Dazu wurden in Versuchskammern unter definierten Bedingungen drei unterschiedliche Alkyd-Farben, die Butanonoxim enthielten, getestet. Unter anderem wurde festgestellt, dass über $68 \%$ des in der aufgetragenen Farbe enthaltenen Butanonoxims nach dem Auftragen in die Innenraumluft gelangen, davon über $90 \%$ innerhalb der ersten $10 \mathrm{~h}$ nach dem Auftragen der Farbe. Die höchste Konzentration in der Innenraumluft hängt vom Butanonoxim-Gehalt in der Farbe ab $[4,5]$.

Zum Vorkommen von Butanonoxim in der Luft von Wohnungen, Kin- 
dergärten, Schulen und Büroräumen in Deutschland liegen wenige Angaben vor. Butanonoxim ist in der Datenbank der Arbeitsgemeinschaft ökologischer Forschungsinstitute e. V (AGÖF) zum Vorkommen von flüchtigen organischen Verbindungen in der Innenraumluft gelistet. Bei 130 meist anlassbezogenen Messungen nach mehr als 8 Stunden ohne Lüftung, von denen nur 13 oberhalb der Bestimmungsgrenze (BG) von $1 \mu \mathrm{g}$ Butanonoxim $/ \mathrm{m}^{3}$ lagen, wurde ein Median von $0,5 \mu \mathrm{g} / \mathrm{m}^{3}$ (halbe BG), ein 95. Perzentil von 3,6 und ein Maximalwert von $17 \mu \mathrm{g} /$ $\mathrm{m}^{3}$ ermittelt [6].

Im Rahmen einer Auswertung von Messungen an Innenraumarbeitsplätzen durch das Institut für Arbeitsschutz der Deutschen Gesetzlichen Unfallversicherung wurden für die Branchengruppe Schule und Verwaltung bei 14 von 39 Messungen aus drei Einrichtungen Konzentrationen oberhalb der Bestimmungsgrenze von $0,4 \mathrm{mg} / \mathrm{m}^{3}$ nach Abdichtarbeiten an Fenstern berichtet [7]. Einen Monat nach der ersten Messung lagen die Messwerte unter der analytischen Bestimmungsgrenze.

\subsection{Lebensmittel, Trinkwasser}

Daten zum Vorkommen von Butanonoxim in Lebensmitteln, Trinkwasser oder anderen Umweltmedien liegen nicht vor.

\subsection{Gesamtexposition/ Innere Exposition}

Da keine Angaben zum Vorkommen von Butanonoxim in anderen Umweltmedien oder Lebensmitteln vorliegen, lässt sich die Gesamtexposition gegenüber Butanonoxim nicht abschätzen.

\section{Toxikokinetik}

In einer Studie mit männlichen und weiblichen trächtigen Mäusen wurde radioaktiv markiertes Butanonoxim nach oraler oder intratrachealer Gabe rasch resorbiert. Eine Exposition gegenüber Butanonoxim führte zu erhöhten Konzentrationen in Leber und im Epithel der Nase unabhängig vom Expositionspfad. Eine erhöhte Radioaktivität wurde auch in Knochenmark, Milz, Speicheldrüsen, Har- derscher Drüse, Darmwand, Brustdrüsen und Pankreas gefunden. In den Feten waren die Gewebekonzentrationen in der Leber am höchsten, im Urin war hohe und im Darm nur minimale Radioaktivität nachweisbar [8].

In einer Studie von Burka et al. [9] wurde männlichen Ratten einmalig eine Mischung aus radioaktiv markiertem und nicht radioaktiv markiertem Butanonoxim oral, intravenös oder dermal in einer Konzentration von 2,7, 27 oder 270 mg/ kg Körpergewicht verabreicht. Butanonoxim wurde vom gastrointestinalen Trakt der Tiere schnell aufgenommen und weitgehend zu $\mathrm{CO}_{2}$ abgebaut. Butanonoxim wurde zu mindestens 5 polaren Metaboliten verstoffwechselt, unter anderem waren Butanon sowie Glucoronid-Konjugate von Butanonoxim nachweisbar, aber nicht alle Metabolite konnten vollständig identifiziert werden. $\mathrm{CO}_{2}$ und Butanon wurden über die Lunge, die Glucoronid-Konjugate und andere polare Metabolite wurden über den Urin ausgeschieden. Die Exkretion über die Faeces betrug weniger als $2 \%$. Weder in den flüchtigen Substanzen (neben $\mathrm{CO}_{2}$ hautsächlich Butanon) noch im Urin konnte Butanonoxim nachgewiesen werden, Butanon dagegen schon. Butanonoxim akkumulierte nicht im Gewebe. Die Autoren vermuten, dass bei einer oralen Gabe von $270 \mathrm{mg}$ Butanonoxim/kg Körpergewicht die Sättigung eines metabolischen Pfades eingetreten sein könnte (bei der Oxidation von Butanon zu CO 2 ) [9].

In weiteren toxikokinetischen Studien wurden für Butanonoxim zwei metabolische Pfade nachgewiesen und die Möglichkeit eines dritten metabolischen Pfades diskutiert. Der bedeutsamste Pfad ist die Hydrolyse von Butanonoxim zu Butanon. Der zweite Pfad ist die Oxidation von Butanonoxim zu Butan-2-nitrat (2-Nitrobutan) mit Hilfe der mikrosomalen Monooxygenase und Folgereaktionen, die zu dem mutagenen Agens Hydroxylaminsulfat führen können. Dieser Pfad ist jedoch quantitativ von relativ geringer Relevanz. Die Möglichkeit eines dritten metabolischen Pfades wird genannt, jedoch nicht näher ausgeführt $[3,10]$.

\section{Wirkungen}

Belastbare Arbeitsplatzstudien oder bevölkerungsbezogene Untersuchungen zur Wirkung eingeatmeten Butanonoxims beim Menschen fehlen. Für die gesundheitliche Bewertung einer inhalativen Exposition gegenüber Butanonoxim werden deshalb Ergebnisse von Untersuchungen an der Maus und Ratte herangezogen.

Ausführlichere Beschreibungen relevanter toxikologischer Studien liegen vor [11-16]. Die folgende Darstellung konzentriert sich auf die für die Ableitung von Innenraumluftrichtwerten maßgeblichen Studien.

\subsection{Subakute, subchronische und chronische Toxizität}

In subakuten Studien wurden F-344-Ratten oder CD-1-Mäuse (jeweils 10 Tiere pro Tierart, Geschlecht und Dosisstufe) gegenüber Butanonoxim über einen Zeitraum von 4 Wochen $(6 \mathrm{~h} / \mathrm{d}, 5 \mathrm{~d} / \mathrm{w})$ exponiert. Bei einer Konzentration von $1450 \mathrm{mg}$ Butanonoxim/ $/ \mathrm{m}^{3}\left(400 \mathrm{ml} / \mathrm{m}^{3}\right)$ wurden in Ratten signifikante hämatologische Veränderungen sowie in beiden Spezies erhöhte Methämoglobinbildung und erhöhte Organgewichte für Leber und Milz festgestellt. Ratten waren die sensitivere Spezies gegenüber der $\mathrm{Bu}$ tanonoxim Inhalation [17]. Darüber hinaus traten in Mäusen nach 4 Wochen degenerative Veränderungen des olfaktorischen Epithels in $20 \%$ der Tiere bei einer Konzentration von 108 mg Butanono$\mathrm{xim} / \mathrm{m}^{3}(30 \mathrm{mg} / \mathrm{ml})$ und in $80 \%$ der Tiere bei einer Konzentration von $360 \mathrm{mg} / \mathrm{m}^{3}$ (100 mg/ml) auf [18].

In einer subchronischen Studie wurden F-344-Ratten (je 10 Tiere pro Geschlecht und Dosisstufe) gegenüber 0, 54, 272 oder $1360 \mathrm{mg}$ Butanonoxim $/ \mathrm{m}^{3}(0,15$, 75 und $375 \mathrm{ml} / \mathrm{m}^{3}$ ) über einen Zeitraum von 3 Monaten $(6 \mathrm{~h} / \mathrm{d}, 5 \mathrm{~d} / \mathrm{w})$ exponiert. Bei einer Konzentration von 1360 mg Butanonoxim $/ \mathrm{m}^{3}$ wurden signifikante hämatologische Veränderungen, eine erhöhte Methämoglobinbildung, erhöhte Körpergewichte (nur männliche Tiere) sowie signifikant erhöhte Organgewichte für Leber und Milz festgestellt [17].

In einer weiteren subchronischen Studie wurden zur Ableitung einer NOAEC 
die Wirkungen von Butanonoxim auf die Degeneration des olfaktorischen Epithels untersucht. Hierzu wurden männliche CD-1-Mäuse (jeweils 10 Tiere pro Dosisstufe) 1, 2, 4 bzw. 13 Wochen gegenüber $0,11,36,108$ oder $360 \mathrm{mg}$ Butanonoxim/ $\mathrm{m}^{3}\left(0,3,10,30,100 \mathrm{ml} / \mathrm{m}^{3}\right)$ exponiert $(6 \mathrm{~h} / \mathrm{d}, 5 \mathrm{~d} / \mathrm{w})$. Nach 13-wöchiger Expositionszeit wurde in der Dosisstufe $36 \mathrm{mg} /$ $\mathrm{m}^{3}$ bei 3 von 10 Tieren eine Degeneration des olfaktorischen Epithels beobachtet. Als subchronische LOAEC und NOAEC wurde von den Autoren eine Konzentration von 36 bzw. 11 mg Butanono$\mathrm{xim} / \mathrm{m}^{3}$ angesehen. Die Inzidenzen für die Degeneration des olfaktorischen Epithels waren bei diesen Konzentrationen in aufsteigender Reihenfolge 0/10 (Kontrolle), $0 / 10,3 / 10,4 / 9$ und 10/10 in den Dosisgruppen. Die Schweregrade der Läsionen betrugen in aufsteigender Reihenfolge der Konzentrationen durchschnittlich $0,0,1,1,75$ und 2,6 auf einer Skala von 0 (nicht vorhanden) bis 5 (schwerwiegend) [18]. Nach Ansicht der Autoren beruhen die festgestellten nasalen Wirkungen weniger auf lokalen, irritativen Effekten, da die Degeneration des olfaktorischen Epithels auch nach oraler Gabe im Tierexperiment beobachtet wurde [13]. Offensichtlich spielt das olfaktorische Epithel bei der Metabolisierung inkorporierten Butanonoxims eine wichtige Rolle [18].

In einer Langzeit-Inhalationsstudie wurden F-344-Ratten und CD-1-Mäuse (jeweils 50 Tiere pro Tierart, Geschlecht und Dosisstufe) gegenüber 0, 54, 272 oder $1360 \mathrm{mg}$ Butanonoxim $/ \mathrm{m}^{3}(0,15$, 75 und $375 \mathrm{ml} / \mathrm{m}^{3}$ ) über einen Zeitraum von 26 Monaten (Ratten) bzw. 18 Monaten (Mäuse) exponiert (6h/d, $5 \mathrm{~d} / \mathrm{w})$ [17]. Dosisabhängig wurden sowohl bei Ratten als auch bei Mäusen Degeneration des olfaktorischen Epithels sowie Lebertoxizität (bei Ratten: spongiose Hepatitis, basophile Foci und Vakuolisierung; bei Mäusen: Pigmentablagerungen, Nekrosen, hepatozelluläre Hypertrophie und granulomatöse Inflammation), Kongestion der Milz, erhöhte Testes- und Milzgewichte, extramedulläre Hämatopoese sowie Kataraktbildung beobachtet. Als sensibelster Wirkendpunkt wurde bei beiden Tierarten eine Degeneration des olfaktorischen Epithels identifiziert, wobei Effekte in der niedrigsten Dosisstufe von 54 mg Butan-

Bundesgesundheitsbl 2015 · 58:505-512 DOI 10.1007/s00103-015-2132-7

(c) Springer-Verlag Berlin Heidelberg 2015

\section{Bekanntmachung des Umweltbundesamtes \\ Richtwerte für Butanonoxim in der Innenraumluft. Mitteilung der Ad-hoc-Arbeitsgruppe Innenraumrichtwerte der Kommission Innenraumlufthygiene und der Obersten Landesgesundheitsbehörden}

\section{Zusammenfassung}

Zum Schutz der Gesundheit der Bevölkerung setzt die Ad-hoc-Arbeitsgruppe Innenraumrichtwerte der Kommission Innenraumlufthygiene und der Obersten Landesgesundheitsbehörden Richtwerte für die Innenraumluft fest. Für eine gesundheitliche Bewertung von Butanonoxim in der Luft liegen keine hinreichend aussagekräftigen Humanstudien vor. In einer gut dokumentierten und als zuverlässig eingestuften chronischen Inhalationsstudie an Ratten und Mäusen wurde eine Degeneration des olfaktorischen Epithels beobachtet, die insbesondere bei Mäusen zu einer konzentrationsabhängigen erhöhten Inzidenz und Ausprägung führten. Aus dieser Studie schätzt die Ad-hoc-Arbeitsgruppe mithilfe einer Benchmarkberechnung eine $B D_{10}$ für kontinuierliche Exposition von
$13,8 \mathrm{mg}$ Butanonoxim $/ \mathrm{m}^{3}$ für die Degeneration des olfaktorischen Epithels ab. Zur Berücksichtigung von Interspezies-Unterschieden wird ein reduzierter Faktor von 1 verwendet, da hinsichtlich des Endpunktes der Mensch nicht empfindlicher erscheint als die Maus. Mit einem Extrapolationsfaktor von 10 für interindividuelle Variabilität sowie einem Faktor von 2 zur Berücksichtigung der im Vergleich mit Erwachsenen höheren Atemrate von Kindern ergibt sich ein Richtwert II (Gefahrenwert) von 0,06 mg Butanonoxim/ $\mathrm{m}^{3}$ und ein Richtwert I (Vorsorgewert) von $0,02 \mathrm{mg}$ Butanonoxim $/ \mathrm{m}^{3}$ Innenraumluft.

Schlüsselwörter

Butanonoxim · Innenraumluft .

Olfaktorisches Epithel · BMD 10 . Richtwert

\section{Indoor air guide values for 2-butanone oxime. Communication from the Ad-hoc Working Group on Indoor Guide Values of the Indoor Air Hygiene Commission and the States' Supreme Health Authorities}

\section{Abstract}

The German Working Group on Indoor Guidelines of the Indoor Air Hygiene Committee and of the Supreme State Health Authorities is issuing indoor air guide values to protect public health. No reliable human studies are available for health evaluation of 2-butanone oxime in indoor air. In a well documented chronic inhalation animal study with rats and mice assessed as reliable, degenerative changes in the olfactory epithelium were observed, which led to a dose related increased incidence and severity, especially in mice. Using a benchmark approach the Working Group assessed a BMD 10 of $13.8 \mathrm{mg} 2$-butanone oxime $/ \mathrm{m}^{3}$ for continuous exposure for the endpoint degeneration of the olfac- tory epithelium. For interspecies differences a reduced factor of 1 was applied due to the same susceptibility of rodents than human for this endpoint. By applying a factor of 10 for interindividual variability, and a factor of 2 to account for the higher respiratory rate of children compared to adults, a health hazard guide value (RW II) of $0.06 \mathrm{mg} 2$-butanone oxime $/ \mathrm{m}^{3}$ indoor air is obtained. A precautionary guide value of $0.02 \mathrm{mg} 2$-butanone oxime $/ \mathrm{m}^{3}$ indoor air is recommended.

\section{Keywords}

2-butanone oxime · Indoor air . Olfactory epithelium $\cdot \mathrm{BMD}_{10} \cdot$ Guide value onoxim $/ \mathrm{m}^{3}$ nur bei Mäusen beobachtet wurden.

In oralen Studien mit subakuter bis subchronischer Exposition von Ratten, Mäusen oder Kaninchen per Schlundsonde oder über das Trinkwasser (Dosisbereich 10-3170 mg Butanonoxim/kg Körpergewicht und Tag) wurden im Wesent- lichen vergleichbare Effekte wie nach inhalativer Exposition beobachtet (Lebertoxizität, Methämoglobinbildung mit Folgewirkungen). In einer 2-Generationenstudie an Ratten sowie in einer Teratogenitätsstudie an Kaninchen war der LOAEL für Methämoglobinbildung übereinstimmend 10 mg Butanonoxim/kg Kör- 
pergewicht und Tag. Bei $200 \mathrm{mg} / \mathrm{kg}$ und Tag traten bei Ratten erhöhte Mortalität und klinische Anzeichen einer Intoxikation (Sedation, Hypoaktivität) auf [19]. In einer Studie mit Verabreichung über das Trinkwasser wurde zusätzlich die auch nach inhalativer Exposition aufgetretene Degeneration des olfaktorischen Epithels beobachtet (LOAEL bei Ratten 175$215 \mathrm{mg} / \mathrm{kg}$ Körpergewicht und Tag, NOAEL 100-120 mg/kg KG und Tag) [13]. In den Studien mit Schlundsondengabe finden sich hierzu keine Angaben. Weiterhin traten in den oberen Dosisgruppen Hyperplasien des Harnblasenepithels auf.

\subsection{Kanzerogenität}

Humandaten zum Vorkommen von Krebserkrankungen nach einer Exposition gegenüber Butanonoxim liegen nicht vor. In den in Abschn. 4.1 dargestellten Langzeitstudien an Maus und Ratte wurde bei männlichen Tieren beider Spezies dosisabhängig eine Zunahme der Inzidenzen an Leberadenomen und -karzinomen beobachtet [17]. Bei männlichen Fischer344-Ratten nahm nach einer Exposition gegenüber 0, 54, 272 oder 1360 mg Butanonoxim $/ \mathrm{m}^{3}\left(0,15,75,375 \mathrm{ml} / \mathrm{m}^{3}\right)$ die Gesamtinzidenz an Leberadenomen und -karzinomen von $0 / 50$ auf $2 / 51,6^{*} / 51$ oder $26^{*} / 51$ signifikant $\left(^{*}\right)$ zu. Bei den weiblichen Tieren beider Spezies stieg die Inzidenz für Leberadenome nicht signifikant an. Leberkarzinome wurden nicht beobachtet. Weiterhin wurde in dieser Studie bei männlichen Ratten eine signifikant erhöhte Inzidenz an Brustdrüsenfibroadenomen für die höchste Dosis beobachtet sowie in je einer Dosisgruppe eine signifikante Abnahme der Inzidenz für Leukämie mononukleärer Zellen für weibliche $\left(272 \mathrm{mg} / \mathrm{m}^{3}\right)$ und männliche $(1360 \mathrm{mg} /$ $\left.\mathrm{m}^{3}\right)$ Ratten.

\subsection{Mutagenität}

Butanonoxim ist kein direktes mutagenes Agens. Der Großteil der Standardtests zeigte weder in vitro mit oder ohne metabolischer Aktivierung noch in vivo eine mutagene oder gentoxische Wirkung auf [11]. Diese negativen Ergebnisse lassen sich dadurch erklären, dass in den meisten nichthepatischen Zellen und unter in vit-
ro-Bedingungen keine Biotransformation des Butanonoxims stattfinden kann.

Jedoch zeigt ein vermuteter reaktiver Metabolit des Butanonoxims, das Nitronat des 2-Nitrobutan, direkte mutagene Wirkung, induzierte DNA-Reparatur und erzeugte in vitro DNA-Modifikationen unter Anwesenheit von Ratten-Arylsulfotransferasen. 2-Nitrobutan selbst wirkt in vitro nicht signifikant mutagen, es wurde jedoch eine starke leberkanzerogene Wirkung nach oraler Gabe in männlichen Ratten beobachtet [20] sowie DNAModifikationen in der Leber nach i.p.-Applikation von 2-Nitrobutan [21, 22].

Folglich zeigten sich nach intraperitonealer Gabe von Butanonoxim der Leber von männlichen Fischer-344-Ratten DNA- und RNA-Modifikationen an Guanosin-Nukleotiden, nach inhalativer Exposition nur RNA-Modifikationen. Vergleichbare DNA-Modifikationen traten auch nach Gabe anderer Ketoxime oder sekundärer Nitroalkane auf. Nach Ansicht der Arbeitsstoff-Kommission der Deutschen Forschungsgemeinschaft deuten die vorliegenden in vivo-Befunde darauf hin, dass es einen gemeinsamen Aktivierungsmechanismus geben könnte, der zu gleichen Nukleinsäure-Modifikationen in der Leber führen könnte [12].

\subsection{Reproduktionstoxizität}

Fruchtbarkeitsstörungen. In den oralen subchronischen Studien an Ratten und Mäusen mit Verabreichung von Butanonoxim über das Trinkwasser (25-335 mg/ kg Körpergewicht und Tag bei Ratten, $110-3170 \mathrm{mg} / \mathrm{kg} \mathrm{KG}$ und Tag bei Mäusen) wurden auch bei der jeweils höchsten Dosis keine histologischen Veränderungen der Reproduktionsorgane, der Spermienqualität oder des weiblichen Zyklus beobachtet [13].

In der bereits beschriebenen LangzeitInhalationsstudie [17] waren die Testesgewichte bei Ratten ab $292 \mathrm{mg} / \mathrm{m}^{3}(75 \mathrm{ml} /$ $\left.\mathrm{m}^{3}\right)$ im Trend und ab $1360\left(375 \mathrm{ml} / \mathrm{m}^{3}\right)$ signifikant erhöht.

Bei einer oralen 2-Generationenstudie an Ratten (Exposition mit 10, 100 und $200 \mathrm{mg}$ Butanonoxim/kg und Tag ab 10 Wochen vor Verpaarung bis zum Ende der Trächtigkeit) war keine Beeinträch- tigung der Fertilität oder Schädigung der Reproduktionsorgane ersichtlich [19].

Entwicklungsstörungen. Es liegen 2 orale Teratogenitätsstudien an weiblichen Ratten (Dosisbereich 25-750 mg/kg KG und Tag, Trächtigkeitstage 6-15) oder Kaninchen (Dosisbereich 10-80 mg/kg KG und Tag, Trächtigkeitstage 6-18) vor [2325]. Hierbei ergab sich ein NOAEL für eine fruchtschädigende Wirkung bei Ratten von $600 \mathrm{mg}$ Butanonoxim/ $\mathrm{kg} \mathrm{KG}$ und Tag, bei Kaninchen von $20 \mathrm{mg} / \mathrm{kg}$ KG und Tag. Diese Dosen wirkten bereits maternal toxisch (Gewichtsreduktion, klinische Symptome, Methämoglobinbildung). Die entsprechende nächst höhere Dosis (Ratte: $750 \mathrm{mg} / \mathrm{kg} \mathrm{KG}$ und Tag, Kaninchen: $40 \mathrm{mg} / \mathrm{kg}$ KG und Tag) wirkte fetotoxisch (verringerte Fetengewichte bei Ratten, erhöhte Abortrate bei Kaninchen), aber nichtteratogen. Bei der o.g. 2-Generationenstudie an Ratten [19] wirkte die höchste Dosis (200 mg Butanonoxim/kg KG und Tag) bereits deutlich toxisch auf die Elterntiere (erhöhte Mortalität, Gewichtsreduktion, klinische Anzeichen von Intoxikation). Postnatale Toxizität (nicht näher spezifiziert) wurde nicht beobachtet.

\subsection{Neurotoxizität}

In einer Studie an Ratten zu neurotoxischen Effekten nach oraler Gabe von 40, 125 oder $400 \mathrm{mg}$ Butanonoxim/kg KG und Tag, $5 \mathrm{~d} / \mathrm{w}, 13$ Wochen) wurden bei der höchsten Dosis vorübergehende verminderte Aktivität und Ataxie nach der Applikation beobachtet. Weitere Effekte (in einer umfangreichen Untersuchung auf Verhaltensveränderungen) oder neuropathologische Auffälligkeiten waren nicht ersichtlich [26]. In anderen vorliegenden Studien wurde ebenfalls eine Dämpfung des Zentralnervensystems beobachtet (Sedation, Aktivitätsminderung, Ataxie; bei inhalativer Exposition ab etwa $2530 \mathrm{mg}$ Butanonoxim/ $/ \mathrm{m}^{3}\left(700 \mathrm{ml} / \mathrm{m}^{3}\right)$, oral ab $200 \mathrm{mg} / \mathrm{kg} \mathrm{KG}$ und Tag).

\subsection{Geruchswahrnehmung}

Daten zur Geruchswahrnehmungsschwelle liegen nicht vor. 
Tab. 1 Berechnete Benchmarkdosen für die Degeneration des olfaktorischen Epithels und die Summe an Adenomen und Karzinomen in der Leber auf Basis der Studien von Newton et al. [17] und [18] mit Hilfe der Software BMDS 2.4 der US-EPA

\begin{tabular}{|llllll}
\hline Endpunkt & Einheit & BMD $_{5}$ & BMDL $_{5}$ & BMD $_{10}$ & BMDL $_{10}$ \\
\hline Degeneration des olfaktorischen Epithels & $\mathrm{mg} / \mathrm{m}^{3}$ & 6,7 & 4,2 & 13,8 \\
\hline Summe Leberadenome und -karzinome & $\mathrm{mg} / \mathrm{m}^{3}$ & 92 & 70 & 187 \\
\hline
\end{tabular}

\section{Bewertung}

Zur gesundheitlichen Wirkung eingeatmeten Butanonoxims liegen keine belastbaren Humandaten vor.

Zur Bewertung der gesundheitlichen Wirkung eingeatmeten Butanonoxims zieht die Ad-hoc-Arbeitsgruppe deshalb Ergebnisse tierexperimenteller Untersuchungen heran $[3,11]$. Die Degeneration des olfaktorischen Epithels in der Maus stellt den empfindlichsten Endpunkt dar. Als niedrigste NOAEC wurde in der subchronischen Studie von Newton et al. [18] eine Konzentration von 11 mg Butanono$\mathrm{xim} / \mathrm{m}^{3}$ ermittelt, als LOAEC für den gleichen Wirkendpunkt $36 \mathrm{mg} / \mathrm{m}^{3}$. Für die Leberkanzerogenität wurde in der chronischen Kanzerogenitätsstudie von Newton et al. [17] eine LOAEC von $1360 \mathrm{mg} /$ $\mathrm{m}^{3}$ identifiziert, Adenome wurden $\mathrm{ab}$ $272 \mathrm{mg} / \mathrm{m}^{3}$ häufiger beobachtet [18].

Zur Bestimmung der Ausgangspunkte (Point of Departure - PoD) wurde für die aus den Studien von Newton et al. [17] und [17, 18] vorliegenden quantalen Daten die Benchmark-Dose-Software (BMDS Version 2.4) der US-amerikanischen Umweltbehörde (EPA) herangezogen. Zur Berücksichtigung der Hintergrundinzidenz wurde die sog. extra riskMethode gewählt. In einem ersten Schritt wurden alle verfügbaren mathematischen Modelle gerechnet, um den Dosis-Wirkungsverlauf zu ermitteln. Nach Prüfung aller zu berücksichtigenden Randbedingungen erwies sich für beide Endpunkte das Quantal-linear-Modell als das Modell mit der besten Kurvenanpassung. Die entsprechenden Bewertungskriterien der US-EPA zeigen, dass die zu Grunde liegenden tierexperimentellen Daten eine sehr gute Eignung für die Verwendung zur Benchmark-Modellierung aufweisen. In - Tab. 1 sind die mit der Methode berechneten jeweiligen Benchmarkdosen dargestellt.

Im Vergleich der Wirkendpunkte „Degeneration olfaktorisches Epithel“ und „Summe Leber-Karzinome/Adenome" ergibt die Berechnung, dass auch die Kombination von Adenomen und Karzinomen zu deutlich höheren BMD-Werten führt als die entsprechenden Benchmarkdosen zu den degenerativen Veränderungen des olfaktorischen Epithels. Leberkarzinome wurden in der Studie von Newton et al. [17] ausschließlich in der höchsten Dosisstufe $\left(1360 \mathrm{mg} / \mathrm{m}^{3}\right)$. Eine Benchmarkberechnung auf Grundlage der nur in dieser einen Dosisstufe beobachteten Leberkarzinome ist nicht möglich.

Insgesamt zeigen die tierexperimentell erhobenen Wirkungsdaten, dass die im Tierversuch bei der höchsten Dosisstufe beobachtete karzinogene Wirkung nicht den sensibelsten Wirkendpunkt darstellt. Im Übrigen kann für eine mögliche kanzerogene Wirkung von Butanonoxim prinzipiell das Vorliegen einer Schwelle unterstellt werden. Diese Schwelle liegt deutlich oberhalb der ermittelten Benchmarkdosen für den betrachteten Wirkendpunkt „Degeneration des olfaktorischen Epithels“.

\subsection{Mechanistische Aspekte zur Kanzerogenität}

Die Studie von Newton et al. [17] deutet daraufhin, dass Butanonoxim als Leberkanzerogen wirken kann. Der Mechanismus ist nicht vollständig aufgeklärt, jedoch lässt sich aus Untersuchungen zu strukturverwandten Verbindungen wie andere Ketoxime oder sekundäre Nitroalkane ein möglicher Aktivierungsmechanismus postulieren. Eine ausführliche Beschreibung der Untersuchungen findet sich in der TRGS 900 „Begründung zu Butanonoxim" [11].

Als mutagenes Agens wird das Hydroxylaminsulfat vermutet. Es kann sich im Verlauf der Biotransformation von $\mathrm{Bu}-$ tanonoxim bilden. Zunächst wird Butanonoxim zu 2-Nitrobutan oxidiert. Dieser Stoffwechselpfad ist in Ratten mit einem
Anteil von $20 \%$ beschrieben [10]. Das als Butan-2-Nitronat vorliegende 2-Nitrobutan wird dann in einer von Aryl-Sulfotransferasen katalysierten Reaktion desoxigeniert und zum Butanonoxim-O-sulfat sulfatiert. Dieses hydrolysiert zu Butanon und Hydroxylamin-O-sulfat. Das Hydroxyl-O-Sulfat setzt spontan hochreaktives Nitreniumion frei, das zur Aminierung von Nukleinsäuren führen kann [27].

Der Einfluss der Arylsulfotransferasen liefert eine mögliche Erklärung für die geschlechtsspezifische Tumorinzidenz. Männliche Ratten weisen eine deutliche höhere Sulfotransferaseaktivität auf als weibliche Ratten und Ratten eine höhere als Mäuse [28]. Die Aktivität der humanen Sulfotransferase 1A1 (SULT1A1) variiert um eine Vielfaches zwischen Individuen, bedingt unter anderem durch Einzelnukleotid-Polymorphismen (SNP) und Kopienanzahlvariationen (CNV) [29].

In der „Begründung zu Butanonoxim“ in der TRGS 900 [11] wird geschlussfolgert, dass eine direkte Interaktion eines leberspezifischen aktivierten Metaboliten mit Nukleinsäuren zur kanzerogenen Wirkung von Butanonoxim beitragen kann. Darüber hinaus spielen auch nicht gentoxisch bedingte lebertoxische Wirkungen bei den beobachteten kanzerogenen Effekten eine Rolle wie z. B. eine proliferative Verstärkung. In der Studie von Newton et al. [17] sind bei Ratten bereits ab der niedrigsten applizierten Dosis von $54 \mathrm{mg}$ Butanonoxim $/ \mathrm{m}^{3}\left(15 \mathrm{ml} / \mathrm{m}^{3}\right)$ lebertoxische Effekte beobachtet worden (Spongiosis Hepatitis, basophile foci, Nodule, Zysten).

\subsection{Bestehende Regelungen}

Im europäischen Gefahrstoffrecht ist $\mathrm{Bu}$ tanonoxim als krebsverdächtig (Karz. 2) eingestuft [1]. Der Ausschuss für Gefahrstoffe (AGS [11]) hat einen Arbeitsplatzgrenzwert von $1 \mathrm{mg}$ Butanonoxim $/ \mathrm{m}^{3}$ auf Grundlage der nicht-kanzerogenen Wir- 
Tab. 2 Derivation of indoor air guide values for butanone oxime: key data

\begin{tabular}{|c|c|c|c|}
\hline Parameter & Value/Descriptor & Dimension & Comments \\
\hline \multicolumn{4}{|l|}{ General Infomartion } \\
\hline CLP Index No & $616-014-00-0$ & & \\
\hline EC No & $202-496-6$ & & \\
\hline CAS No & $96-29-7$ & & \\
\hline CLP CMR Classification & Carc. 2 & & EC No 1272/2008 Annex VI \\
\hline Indoor Air Guide Value Status & Final & & \\
\hline Guide Value II & 0.06 & $\mathrm{mg} / \mathrm{m}^{3}$ & \\
\hline Guide Value I & 0.02 & $\mathrm{mg} / \mathrm{m}^{3}$ & \\
\hline Conversion factor $1 \mathrm{ml} / \mathrm{m}^{3}=$ & 3.62 & $\mathrm{mg} / \mathrm{m}^{3}$ & \\
\hline Year & 2014 & & \\
\hline \multicolumn{4}{|l|}{ Database } \\
\hline Key study & Newton et al. [18] & & $\begin{array}{l}\text { Newton et al. Inhalation To- } \\
\text { xicology 14: 1249-1260 }\end{array}$ \\
\hline Species & CD1-mice & & Male \\
\hline Route/Type of Study & Inhalation & & Whole body \\
\hline Study length & Subchronic & & 13 weeks \\
\hline Inhalative exposure duration & $6 \mathrm{~h} / \mathrm{d}, 5 \mathrm{~d} / \mathrm{w}$ & & \\
\hline Critical endpoint & Olfactory degener & & \\
\hline POD & $\mathrm{BMD}_{10}$ & & $\begin{array}{l}10 \text { animals/dose, BMDS } \\
\text { Version 2.4 US-EPA (Dicho- } \\
\text { tomous data, Extra Risk } \\
\text { method, BMR05, Restricted } \\
\text { parameters, Quantal linear } \\
\text { model) }\end{array}$ \\
\hline
\end{tabular}

\begin{tabular}{|c|c|c|c|}
\hline POD & 13.8 & $\mathrm{mg} / \mathrm{m}^{3}$ & \\
\hline \multicolumn{4}{|l|}{ Assessment factors } \\
\hline $\begin{array}{l}\text { Dose - response assessment } \\
\text { factor }\end{array}$ & n.a. & & \\
\hline $\begin{array}{l}\text { Adjusted exposure duration } \\
\text { factor (time scaling) }\end{array}$ & 5.6 & & $\begin{array}{l}6 \mathrm{~h} / \mathrm{d}, 5 \mathrm{~d} / \mathrm{w} \text { to } 24 \mathrm{~h} / \mathrm{d} \text {, } \\
7 \mathrm{~d} / \mathrm{w}\end{array}$ \\
\hline Adjusted study length factor & 2 & & Subchronic-chronic \\
\hline $\begin{array}{l}\text { Route-to-route extrapolation } \\
\text { factor }\end{array}$ & n.a. & & \\
\hline $\begin{array}{l}\text { Adjusted absorption factor } \\
\text { (inhalative/oral) }\end{array}$ & n.a. & & \\
\hline \multirow[t]{2}{*}{ Interspecies factors } & 1 & & Kinetic \\
\hline & 1 & & $\begin{array}{l}\text { Dynamic (standard } 2.5 ; \\
\text { reduction to } 1 \text { reasonable } \\
\text { due to higher susceptibility } \\
\text { of rodents than human) }\end{array}$ \\
\hline Intraspecies factor & 10 & & General population \\
\hline Sensitive population factor & 2 & & Insufficient data in children \\
\hline $\begin{array}{l}\text { Other adjustment factors } \\
\text { Quality of whole databse }\end{array}$ & n.a. & & \\
\hline \multicolumn{4}{|l|}{ Result } \\
\hline Total assessment Factor (TAF) & 224 & & \\
\hline $\mathrm{POD} / \mathrm{TAF}$ & 0.06 & $\mathrm{mg} / \mathrm{m}^{3}$ & $\begin{array}{l}\text { Rounded guide value II: } \\
0.06 \mathrm{mg} / \mathrm{m}^{3}\end{array}$ \\
\hline $\mathrm{BMDL}_{5} / \mathrm{TAF}$ & 0.02 & $\mathrm{mg} / \mathrm{m}^{3}$ & $\begin{array}{l}\text { Rounded guide value } \mathrm{I}: \\
0.02 \mathrm{mg} / \mathrm{m}^{3}\end{array}$ \\
\hline
\end{tabular}

kungen abgeleitet. Basis bildete die Studie von Newton et al. [17]. Mittels eines Benchmark-Verfahrens (BMDS $2.2 \mathrm{der}$ US-EPA) wurde eine $\mathrm{BMD}_{5}$ ermittelt und gemäß Methodik die Extrapolationsfaktoren für humanäquivalente Atemkonzentration von 2 und Intraspezies- Variabilität von 5 verwendet.

\subsection{Ableitung von Richtwerten für 2-Butanonoxim in der Innenraumluft}

Belastbare Kenntnisse zur gesundheitlichen Wirkung eingeatmeten Butanonoxims beim Menschen fehlen. Die Ableitung von Richtwerten stützt sich deshalb auf die Ergebnisse tierexperimenteller Untersuchungen. Als kritischer Endpunkt wird die lokal schädigende Wirkung von Butanonoxim auf das olfaktorische Epithel der Ratte und der Maus angesehen [17, 18]. Die Degeneration des olfaktorischen Epithels war bei der Maus stärker ausgeprägt. Die Ad-hoc-Arbeitsgruppe Innenraumrichtwerte zieht deshalb die Studien an der Maus zur Ableitung von Richtwerten heran.

Richtwert II. Nach dem Basisschema [3] ist für die Festsetzung von Richtwerten für die Innenraumluft von der niedrigsten (beobachteten) nachteiligen Wirkungskonzentration auszugehen. Falls sich der zugrunde liegende Datensatz für eine Benchmarkdosis-Abschätzung eignet, kann auch dieses Verfahren herangezogen werden. Die Ad-hoc-Arbeitsgruppe beschließt, in Anlehnung an die Vorgehensweise der HumanbiomonitoringKommission des Umweltbundesamtes [30] als Ausgangspunkt für die Ableitung eines Richtwertes II für Butanonoxim in der Innenraumluft von der $\mathrm{BMD}_{10}$ auszugehen. Als kritische Studie zieht die Adhoc-Arbeitsgruppe die subchronische Inhalationsstudie von Newton et al. [18] heran.

Für die Ableitung des Richtwertes II werden folgende Extrapolationsfaktoren verwendet:

- Für die Extrapolation von einer subchronischen auf eine chronische Exposition ein Faktor von 2.

- Für die zeitliche Anpassung von 6 auf $24 \mathrm{~h}$ und von 5 auf 7 Tage ein Faktor von $24 / 6^{\star} 7 / 5=5,6$.

- Zur Berücksichtigung von Interspezies-Unterschieden erscheint ein Faktor 1 angemessen, da hinsichtlich des Endpunktes Degeneration des olfaktorischen Epithels der Mensch nicht empfindlicher erscheint als die Maus. 
- Die interindividuelle Variabilität der Bevölkerung wird mit einem Faktor 10 berücksichtigt.

- Nach dem Basisschema [3] ist zu erörtern, ob ein zusätzlicher Faktor zum Schutz von Kindern erforderlich ist. Angesichts der im Vergleich zu Erwachsenen höheren Atemrate pro kg Körpergewicht von Kindern hält die Ad-hoc-Arbeitsgruppe die Verwendung eines zusätzlichen Kinderfaktors von 2 hier für angemessen.

Damit ergibt sich als Richtwert II: $13,8 \mathrm{mg} /$ $\mathrm{m}^{3}:[2 \times 5,6 \times 1 \times 10 \times 2]=0,06 \mathrm{mg} / \mathrm{m}^{3}$. Die Ad-hoc-Arbeitsgruppe legt als Richtwert II $0,06 \mathrm{mg}$ Butanonoxim $/ \mathrm{m}^{3}$ fest.

Richtwert I. Für die Ableitung des Richtwertes I geht die Ad-hoc-Arbeitsgruppe gemäß Basisschema von der subchronischen $\mathrm{BMDL}_{5}$ von 4,2 mg Butanonoxim/ $\mathrm{m}^{3}$ aus. Es werde dieselben Extrapolationsfaktoren verwendet wie für die $\mathrm{Ab}$ leitung des Richtwertes II. Somit ergibt sich als Richtwert I: $4,2 \mathrm{mg} / \mathrm{m}^{3}$ : [2×5,6× $1 \times 10 \times 2]=0,02 \mathrm{mg} / \mathrm{m}^{3}$. Die Ad-hoc-Arbeitsgruppe legt als Richtwert I 0,02 mg Butanonoxim $/ \mathrm{m}^{3}$ fest.

Eine Zusammenfassung der wesentlichen Schritte zur Ableitung von Richtwerten für Butanonoxim in der Innenraumluft gibt $\bullet$ Tab. 2 .

\section{Anmerkungen}

Der Text dieser Empfehlung wurde von Dr. Susanne Rudzok, Herrn Dirk Heller, Frau Silvia Sievering und Dr. Martin Kraft mit Beiträgen von Dr. Helmut Sagunski erarbeitet und von der Ad-hoc-Arbeitsgruppe Innenraumrichtwerte im November 2014 verabschiedet. Die Literaturrecherche wurde im Februar 2014 abgeschlossen.

Einzelne Textteile wurden dem Ausschuss für Gefahrstoffe (AGS) im Vorfeld seiner im Juli 2013 in TRGS 900 veröffentlichten Begründung zu Butanonoxim [11] freundlicherweise zur Verfügung gestellt. Die Berechnung der PoDs für Butanonoxim der Ad-hoc-Arbeitsgruppe Innenraumrichtwerte führte zu identischen Ergebnissen (BMD/BMDL) wie in der vom AGS veröffentlichten Begründung zu Butanonoxim. Detaillierte
Übersichten zu den Inzidenzen der verschiedenen Endpunkte sowie den graphischen und statistischen Auswertungen der Benchmark- Modellierung finden sich in der Veröffentlichung des AGS. Identische Textpassagen in der hier vorliegenden Fassung sowie in der Begründung des AGS stammen von den hier genannten Personen.

\section{Einhaltung ethischer Richtlinien}

Interessenkonflikt. Die Autoren geben an, dass kein Interessenkonflikt besteht.

\section{Literatur}

1. GHS (2008) Verordnung (EG) Nr. 1272/2008 des Europäischen Parlaments und des Rates vom 16. Dezember 2008 über die Einstufung, Kennzeichnung und Verpackung von Stoffen und Gemischen, zur Änderung und Aufhebung der Richtlinien 67/548/EWG und 1999/45/EG und zur Änderung der Verordnung (EG) Nr. 1907/2006 (ABI. L 353 31.12.2008S.1), Anhang VI, Tab. 3.1

2. Ad-hoc-Arbeitsgruppe Innenraumrichtwerte (2012) Richtwerte für die Innenraumluft: erste Fortschreibung des Basisschemas. Bundesgesundheitsbl 55:279-290

3. Environment Canada (2010). Screening Assessment for the Challenge. 2-Butanone, oxime (Butanone oxime). Environment Canada, Health Canada, March 2010. http://www.ec.gc.ca/substances/ ese/eng/challenge/batch7/batch7_96-29-7.cfm. Zugegriffen: Dez. 2011

4. EPA (2002) Inside IAQ, EPA's indoor air quality research update. Environmental Protection Agency. EPA/000/X-00/000 Fall/Winter 2002. Zugegriffen: 1. Juni 2012

5. Chang JCS, Guo Z, Sparks LE (1998) Exposure and emission evaluations of methyl ethyl ketoxime (MEKO) in alkyd paints. Indoor Air 8:295-300. (ISSN 0905-6947)

6. Hofmann H, Plieninger P (2008) Bereitstellung einer Datenbank zum Vorkommen von flüchtigen organischen Verbindungen in der Raumluft. Forschungsbericht 20561243 . Arbeitsgemeinschaft ökologischer Forschungsinstitute (AGÖF) e. V., im Auftrag des Umweltbundesamts. www.umweltdaten.de/publikationen/fpdf-I/3637.pdf. Zugegriffen: 1. Juni 2012

7. IFA (2012) GESTIS-Stoffdatenbank. Gefahrstoffinformationssystem der Deutschen Gesetzlichen Unfallversicherung.,MEGA-Auswertungen zur Exposition gegenüber 2-Butanonoxim". Institut für Arbeitsschutz der Deutschen Gesetzlichen Unfallversicherung. http://www.dguv.de/medien/ifa/de/ fac/reach/mega_auswertungen/butanonoxim_d. pdf. Zugegriffen: 01. Juni 2012

8. DFG (2011) MAK- und BAT-Werte-Liste 2011. Deutsche Forschungsgemeinschaft. Senatskommission zur Prüfung gesundheitsschädlicher Arbeitsstoffe. Mitteilung 47. WILEY-VCH Verlag GmbH, Weinheim

9. Burka LT, Black SR, Mathews JM (1998) Disposition of methyl ethyl ketoxime in the rat after oral, intravenous and dermal administration. Xenobiotica 28(19):1005-1015
10. Janku SE, Faller TH, Dekant W, Csanády GA, Filser JG (2000) Inhalation kinetics of methyl ethyl ketoxime in male and female rats: differentiation between three pathways. Abstract 237. Toxicol Lett 116(Suppl 1):64-65

11. AGS (2013) Begründung zu Butanonoxim in TRGS 900. Ausschuss für Gefahrstoffe. http://www.baua. de/de/Themen-von-A-Z/Gefahrstoffe/TRGS/pdf/ 900/900-butanonoxim.pdf?_blob=publicationFile $\& v=3$. Zugegriffen: 1. Feb 2014

12. Henschler D, Greim H (Hrsg) (1997) Gesundheitsschädliche Arbeitsstoffe: toxikologisch-arbeitsmedizinische Begründungen von MAK-Werten. Weinheim: VCH Loseblattsammlung. 24. Lfg.

13. US-NTP (1999) Technical report on the toxicity studies of methyl ethyl ketoxime (CAS No. 96-297). Administered in drinking water to $\mathrm{F} 344 / \mathrm{N}$ rats B6C3F1 Mice. National Toxicology Program report 51, Juli 1999. http://toxnet.nlm.nih.gov/cgi-bin/ sis/search/a?dbs+hsdb:@term+@DOCNO+8043. Zugegriffen: 1. Juni 2012

14. EPA (2003) Toxicological review of methyl ethyl ketone (CAS No. 78-93-3). In Support of Summary Information on the Integrated Risk Information System (IRIS) September 2003 U.S. Environmental Protection Agency, Washington DC. http://www. epa.gov/iris/subst/0071.htm. Zugegriffen: 1. Juni 2012

15. Schneider K, Schuhmacher-Wolz U, Oltmanns J, Hassauer M (2002) Klassierung krebserzeugender Stoffe zur Begrenzung der Massenkonzentration im Abgas nach der Nr. 5.2.7.1.1 der TA Luft-Novelle (Entwurf)/Umweltbundesamt. Berlin: Erich Schmidt Verlag. (Umweltbundesamt-Berichte; 2002-2003, ISBN 3-503-06633-0)

16. Schuhmacher-Wolz U, Konietzka R, Schneider K (2002) Using carcinogenic potency ranking to assign air contaminants to emission classes. Regulat Toxicol Pharmacol 36:221-233

17. Newton PE, Wooding WL, Bolte HF, Derelanko MJ, Hardisty JF, Rinehart, WE (2001) A chronic inhalation toxicity/oncogenicity study of methylethylketoxime in rats and mice. Inhal Toxicol 13:10931116

18. Newton PE, Bolte HF, Derelanko MJ, Hardisty JF, Rinehart WE (2002) An evaluation of changes and recovery in the olfactory epithelium in mice after inhalation exposure to methylethylketoxime. Inhal Toxicol 14:1249-1260

19. Tyl RW, Gerhart JM, Myers CB et al (1996) Reproductive toxicity evaluation of methylethyl ketoxime by gavage in $\mathrm{CD}$ rats. Fund Appl Toxicol 31:149-161

20. Fiala ES, Sodum RS, Hussain NS, Rivenson A, Dolan $L$ (1995) Secondary nitroalkanes: Induction of DNA repair in rat hepatocytes, activation by aryl sulfotransferase and hepatocarcinogenicity of 2-nitrobutane and 3-nitropentane in male F344 rats. Toxicol 99:89-97

21. Hussain NS, Nie G, Conaway CC, Sohn OS, Fiala ES (1991) Oxidative damage to liver DNA and RNA of rats treated with ketoximes. Proc Am Assoc Canc Res 32:149

22. Conaway CC, Nie G, Hussain NS, Fiala ES (1991) Comparison of oxidative damage to rat liver DNA and RNA by primary nitroalkanes, secondary nitroalkanes, cyclopentanone oxime, and related compounds. Canc Res 51:3143-3147

23. IHF (1990a) Range-finding teratology study in rabbits with MEKO. Springborn Laboratories Inc. 3224.2. Industrial Health Foundation. NTIS OTS 529841 
24. IHF (1990b) Range-finding teratology study in rats. Springborn Laboratories Inc. 3224.1. Industrial Health Foundation. NTIS OTS 529841

25. Mercieca MD, Rinehart WE, Hodgson JR, Derelanko MJ (1991) Developmental toxicity study of methyl ethyl ketoxime (MEKO) in two species. Teratol 43:454-455

26. Schulze GE, Derelanko MJ (1993) Assessing the neurotoxic potential of methyl ethyl ketoxime in rats. Fund Appl Toxicol 21:476-485

27. Sodum RS, Sohn OS, Nie G, Fiala ES (1994) Activation of the liver carcinogen 2-nitropropane by aryl sulfotransferase. Chem Res Toxicol 7:344-351

28. Okuda H, Nojima H, Watanabe N, Watabe T (1989) Sulphotransferase-mediated activation of the carcinogen 5-hydroxymethyl-chrysene. Species and sex differences in tissue distribution of the enzyme activity and a possible participation of hydroxysteroid sulphotransferases. Biochem Pharmacol 38 (18):3003-3009

29. Hildebrandt M, Adjei A, Weinshilboum R et al (2009) Very important pharmacogene summary: sulfotransferase 1A1. Pharmacogenet Genomics 19:404-406

30. HBM-Kommission (2014) Grundsatzpapier zur Ableitung von HBM-Werten. Stellungnahme der Kommission Human-Biomonitoring des Umweltbundesamtes. Bundesgesundheitsbl 57:138-147 